
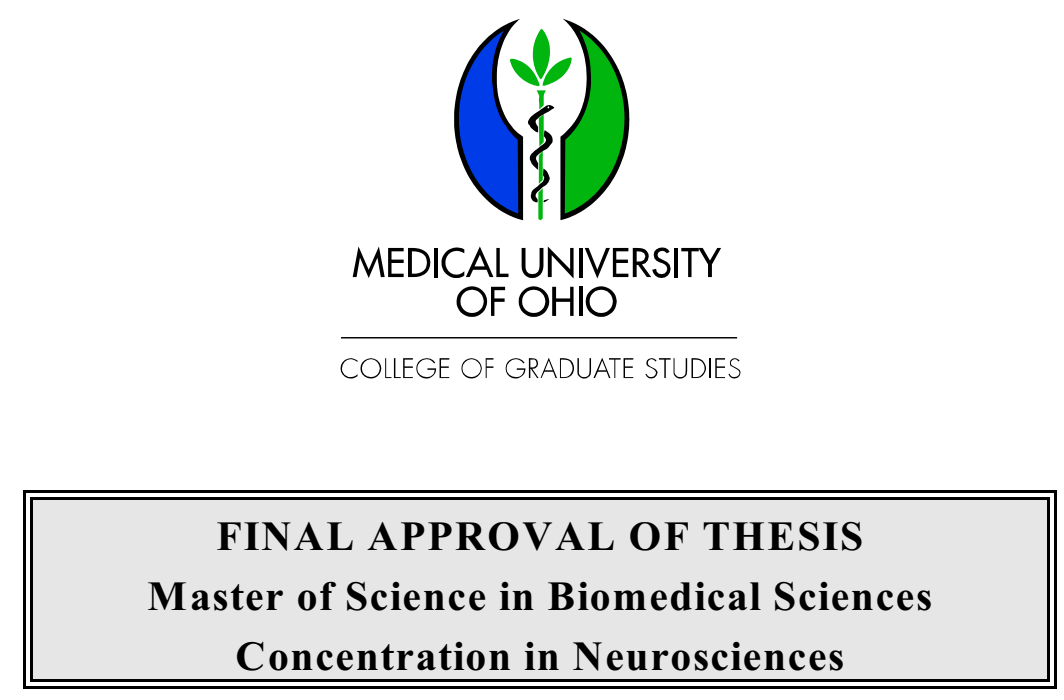

The Effects of Elevated Serotonin Levels on Patterns of GAP-43 Expression During Barrel Development in Rat Somatosensory Cortex

\author{
Submitted by \\ Kay Kesterson \\ In partial fulfillment of the requirements for the degree of \\ Master of Science in Biomedical Sciences \\ Date of Defense: \\ May 3, 2005 \\ Major Advisor \\ Robert Rhoades, Ph.D. \\ Academic Advisory Committee \\ Richard Lane, Ph.D. \\ Carol Bennett-Clarke, Ph.D. \\ Nicolas Chiaia, Ph.D. \\ Donald Godfrey, Ph.D. \\ Dean, College of Graduate Studies \\ Keith K. Schlender, Ph.D.
}


Effects of Elevated Serotonin Levels on Patterns of GAP-43 Expression During

Barrel Development in Rat Somatosensory Cortex

Kay Lee Kesterson

Medical College of Ohio

2005 


\section{DEDICATION}

I dedicate this thesis to my parents, Henry and Tuhu chi Kesterson, who have loved me unconditionally and taught me to pursue knowledge with diligence and to my husband, Matthew David Smith, who has stood by me with love during the worst and best moments of my academic pursuits. 


\section{ACKNOWLEDGEMENTS}

I would like to gratefully thank Rebecca Wynn and Asad Shah for assistance with injections. I would also like to thank Rebecca Wynn and Dawn Pauli for technical assistance. Finally, I would like to thank Dr. Robert Rhoades and Dr. Richard Lane for their intellectual advisement on the design and execution of the experiments and for their review and critique of my manuscript. 


\section{TABLE OF CONTENTS}

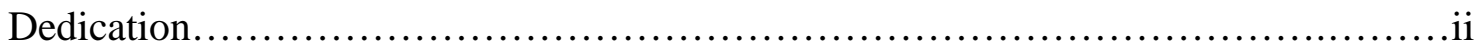

Acknowledgements.........................................................

Table of Contents.............................................................

Introduction................................................................

Literature ........ $\quad$....................................................

Manuscript 1:

Effects of Elevated Serotonin Levels on Patterns of GAP-43 Expression During Barrel Development in Rat Somatosensory Cortex..................................10

References............................................................... 37

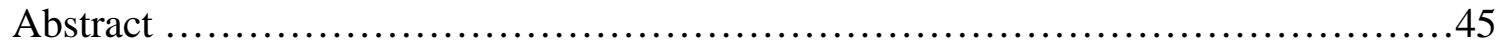




\section{INTRODUCTION}

Several studies in the early 1900s observed cytoarchitectonic irregularities in layer IV of the cerebral cortex of various rodents. In the late 1960s, Woolsey (1967) found that these irregularities partially coincided with the primary somatosensory cortex (SI) as defined by the low-resolution evoked potential technique. Subsequent research by Woolsey and Van der Loos (1970) demonstrated that Nissl-stained sections cut parallel to the overlying pial surface of the cortex displayed an array of cylindrical or oval-shaped multineuronal units which they called "barrels." Further research revealed a morphological and functional correlation between the barrels and the mystacial vibrissae of the rat and mouse and that a critical period for the establishment of the characteristic vibrissae-related barrels during postnatal development existed. These discoveries established barrel structure as one of the foremost studied models for investigating the structure, function, plasticity and development of somatosensory systems.

Every cortical sensory field is the end point of multiple thalamic pathways (Macchi, 1983). Because it is easy to visualize the segregation of various ascending sensory pathways in the thalamus and the barrel cortex, the barrel field of the rodent cortex provides an observable model for the integration of multiple thalamic inputs. The thalamic nuclei that project to the barrel cortex are divided into two functional categories: the primary somatosensory and the secondary somatosensory pathway. The research in this paper is mainly concerned with the primary somatosensory pathway of multiple axons from the thalamic ventral posterior nucleus (VP) that target the layer IV barrels and 
the secondary somatosensory pathway of the axons from the rostral section of the thalamic posterior complex (POm) that terminate in the interbarrel space between the barrels of layer IV of the SI, namely the septa.

The mystacial-related barrel field also provides researchers with a model for examining the role of neurotransmitters and their receptors in developmental neurobiology. Synaptic connections in the rat and mouse cortex are generally completed by postnatal week 3 or 4 . During the postnatal weeks 1-4, thalamocortical, callosal and intrinsic connections are defined, sharpened and refined. During this time period, the presence or absence and the concentration of certain neurotransmitters and their receptors can alter the normal formation of vibrissae-related patterns in the VP and layer IV of the SI as demonstrated by genetic and pharmacological manipulation. Although many neurotransmitters have been implicated in somatosensory development, the role of serotonin (5-HT) in barrel development has been studied considerably due to welldefined transient expression of a barrel pattern in the SI during initial barrel formation. Furthermore, 5-HT has also been shown to play an important role in influencing neuronal differentiation in embryonic and early postnatal development (Lauder et al.,1983) has been studied extensively. Finally, the transient expression of 5-HT during the first two postnatal weeks (D’Amato, 1987) indicates a strong role in the projection of thalamocortical axons to layer IV of the SI and thus, barrel pattern formation.

Growth associated molecules have also demonstrated a vibrissae-related pattern in layer IV of the cortex. Several studies have proposed that GAP-43, a nervous systemspecific phosphoprotein that binds to the plasma membrane of growth cones, is 
implicated in the preliminary ordering and segregation of thalamocortical axons. Gap-43 is transiently expressed in a vibrissae-related pattern in layer IV of the S-I during the first postnatal week. The GAP-43 expression in the barrels of layer IV of the SI disappears by P-8; however, the expression increases in the septal area between the barrels.

In the late 1990s, studies in our lab revealed that significantly elevated levels of 5HT pharmacologically induced by clorgyline, a monoamine oxidase A (MAO-A) inhibitor, during the first postnatal week disrupts the normal barrel pattern formation (Boylan et al., 2000b). The disruption yields a "barrelless" morphology. Surprisingly, the "barrelless" vibrissae-related pattern does not persist into the second week. By P-8, normal segmentation of the barrels returns. It was speculated that the high levels of 5-HT would also modulate the expression of other neuronal molecules in layer IV of the SI. If 5-HT affected other molecules, would the affect be similar or different? Which molecules would be affected?

Since the transient barrel pattern reflective of GAP-43 immunoreactivity is similar to the time course of 5-HT expression and coincides with the appearance of thalamocortical axons, it is hypothesized that the GAP-43 vibrissae-related pattern might also be affected by elevated levels of 5-HT. The disappearance of the GAP-43 barrel pattern and the emergence of increase in staining in the septa around the barrels by P-8 might be reflective of the reformation of the barrel pattern observed by Boylan et al. (2000b). Furthermore, Clarke et al. (1996) demonstrated that 5-HT affected GAP-43 immunoreactivity in astroglial cells and Ching et al. ( 1994) showed that GAP-43 was present in all areas of the spinal cord that showed the expression of 5-HT suggesting that 
there is an interaction between 5-HT and GAP-43. Thus, the purpose of this thesis is to determine if high levels of serotonin affect the expression of GAP-43 in the SI. 


\section{LITERATURE REVIEW}

\section{$\underline{5-H T}$ and Several of its Receptor Subtypes in the Developing SI}

Several studies have demonstrated dense 5-HT immunoreactivity in the developing cortices of rodents that closely matches the thalamocortical pattern for thalamocortical afferents growing into the SI (D’Amato et al., 1987; Fujimiya et al. 1986; Rhoades et al., 1990) a vibrissae-related pattern in layer IV of the SI. However, 5-HT immunoreactivity is not contained in the thalamocortical axons (Rhoades et al., 1990), but rather this immunoreactivity is contained in the serotonergic axons arising from the nucleus raphe dorsalis (Bennett-Clarke et al., 1991) during the first two postnatal weeks of the rodent.

Recent studies have also demonstrated that three 5-HT related receptor subtypes transiently express a thalamocortical axon pattern in layer IV of the SI. Serotonin 1B receptors $\left(5-\mathrm{HT}_{1 \mathrm{~B}}\right)($ Bennett-Clarke, 1993; Leslie et al., 1992) transiently appear in a mystacial vibrissae-related pattern in the somatosensory cortex (S-I) during the first 2 postnatal weeks (Chiaia et al., 1994; D’Amato,1987; Fujimiya et al., 1986;Lebrand et al., 1996; Rhoades et al., 1990). These receptors are located primarily on thalamocortical axon terminals (Bennett-Clarke et al., 1993). Secondly, vibrissae-related thalamocortical axons and their parent cells also transiently express the high affinity 5-HT transporter (SERT) for the first 2 postnatal weeks, allowing 5-HT uptake into these fibers during this time period (Bennett-Clarke, 1996; Boylan et al., 2000a; Bruning and Liangos, 1997; Lebrand et al., 1996; Mansour-Robaey et al, 1998). Finally, serotonin 2A (5- $\left.\mathrm{HT}_{1 \mathrm{~B}}\right)$ 
(Mansour-Robaey et al, 1998) was shown to assume a vibrissae-related pattern in lamina IV of the developing SI. However, the elements that express these receptors is unknown and the vibrissae-related pattern of the 5-HT $1 \mathrm{~B}$ does not occur until P-7 or 8.

\section{Modulation of Cortical Development by Elevated Levels of 5-HT}

Previous research has demonstrated that altering cortical 5-HT concentrations during perinatal development as a result of pharmacological or genetic manipulations disrupts the vibrissae-related pattern in the rodent S-I. A transgenic mouse line (Tg8) lacking the gene that encodes monoamine oxidase $\mathrm{A}\left(\mathrm{MAO}_{\mathrm{A}}\right)$ have significantly elevated 5-HT levels and a complete absence of vibrissae related barrels in the S-I (Cases et al., 1996). A 700-900\% increase in the amount of 5-HT is present in Tg8 pups during the first postnatal week compared to the normal $(\mathrm{C} 3 \mathrm{H})$ mice. By the end of postnatal week 2, 5-HT levels return to normal (Cases et al., 1995). In 1996, Cases et al. demonstrated a permanent disorganization of the barrel field (PMBSF) in tangential cortical sections as revealed by cytochrome oxidase histochemistry, a marker of cortical cells, in Tg8 pups and adults compared to the normal barrels observed in layer IV of the SI in normal pups and adults. The appearance of the PMBSF was blurry. Furthermore, glial cortical cells in the septa were affected by the 5-HT elevations demonstrated by a completely absent tenascin immunoreactivity in the MAO-A knockout P8 pups. In the cortex of Tg8 pups, 5-HT immunostaining demonstrated intense staining in the PMBSF with no clear delineation between the barrels and the septa indicating an alteration in thalamic afferents. In order to determine that the increased 5-HT concentration definitely were the 
cause of these abnormalities observed in the PMBSF of layer IV of the SI, parachlorophenylalanine (PCPA) was injected into the Tg8 and the C3H pups to reduce the concentration of 5-HT. Normal pups revealed normal CO histochemistry and absent 5-HT immunostaining as expected. In MAO-A knockouts, 5-HT and tenascin immunohistochemistry and CO histochemistry revealed a recovered barrel pattern. Alternatively, pharmacological elevation of 5-HT in normal mice was induced by the admistration of clorgyline, an MAO-A inhibitor during the first postnatal week. 5-HT immunostaining revealed a partial blurring of the PMBSF that persisted into adulthood. Further investigation with the Tg8 mice revealed a critical time period existed that would alter the barrel field with elevated levels of 5-HT (Vitalis et al., 1998). Administration of clorgyline between embryonic day 15 (E15) and P7 would disrupt barrel formation in the PMBSF. However, clorgyline injections during embryonic life only or starting on P-4 did not disrupt the barrel pattern.

Pharmacological elevation of cortical 5-HT with clorgyline in rat pups yields a dose-dependent range of disruptions in thalamocortical development (Kesterson et al., 2005). Staining of layer IV aggregates with CO and Nissl staining, labeling of vibrissaerelated thalamocortical afferents with 1,1'-dioctadecyl-3,3,3”,3'tetramethylindocarbocyanine perchlorate (DiI) and 5-HT immunostaining were altered with significantly elevated levels of 5-HT during the first postnatal week. The severity of alterations increased with more injections of clorgyline. Perinatal rodents treated with clorgyline (10 mg/kg) two and three times daily yielded larger vibrissae-related barrels and higher cortical 5-HT levels revealed by high performance liquid chromatography 
(HPLC) compared to control animals. Four daily clorgyline injections produced significantly larger vibrissae-related barrels with indistinct septa in the S-I and significantly augmented cortical 5-HT levels (Kesterson et al., 2005). Increasing clorgyline injections to five times daily yields an intense "barrelless" pattern in the S-I devoid of any normal segmentation and delineation between the barrels and the septa. Cortical 5-HT levels were 1500\% above normal levels in rats killed on postnatal day (P-) 6 (Boylan et al., 2000b). However, while the disorganization in rats is more profound than in mice, the alterations in rats are not permanent. By P-8, thalamocortical axons and layer IV cells begin to reorganize and form vibrissae-related patterns despite continued elevation of cortical 5-HT levels. By P-10, thalamocortical axons and layer IV aggregates form qualitatively and quantitatively normal patches corresponding to the mystacial vibrissae (Boylan et al., 2000b; Kesterson et al., 2005).

\section{A Possible Role for GAP-43 in Thalamocortical Axon Pathfinding}

GAP-43, a nervous system-specific phosphoprotein that binds to the plasma membrane of growth cones, is suggested to be involved in the preliminary ordering and segregation of thalamocortical axons (Erzurumlu et al., 1990; Maier et al., 1999). In the developing S-I, GAP-43 is transiently expressed as a vibrissae-related pattern in the cortex from P-3 to P-7 demonstrating a temporal coincidence with the time-course of thalamocortical axon segregation and barrel formation in layer IV of the SI in normal rats. After P-7, the GAP-43-positive patches in S-I disappear and the density of staining is increased in the septa between the barrels. This complementary pattern of GAP-43 
permanently persists into adulthood (Erzurumlu et al., 1990). In mice lacking the gene encoding GAP-43, thalamocortical axons do not extend to layer IV of S-I, and an ordered whisker-related somatotopic map never forms (Maier et al., 1999) strongly suggesting that the role of GAP-43 is important in thalamocortical axon pathfinding.

\section{Interactions Between GAP-43 and 5-HT in the Central Nervous System}

Studies have revealed that GAP-43 may be co-localized with serotonergic neurons strongly suggesting an interaction between them. In situ hybridization techniques revealed hybridization signals of GAP-43 mRNA and tryptophan hydroxylase mRNA overlap each other in the nucleus centralis superior (NCS) and the nucleus raphe dorsalis (NDR) in the brainstem of adult rats. The intracerbroventricular administration of the neurotoxin, 5,7-dihydroxytryptamine (5,7-DHT) to rats lead to a loss of the GAP-43 hybridization signal indicating that high levels of GAP-43 are synthesized in serotonergic neurons of the raphe nuclei (Bendotti et al., 1991). Double-labeling immunofluorescence of 5-HT and GAP-43 revealed that GAP-43 immunostaining was present in all spinal cord regions that contained 5-HT immunoreactivity (Ching et al., 1994). 


\title{
Manuscript \#1
}

\section{Effects of Elevated Serotonin Levels on Patterns of GAP-43 Expression During Barrel Development in Rat Somatosensory Cortex}

\author{
Kay L. Kesterson* \\ Richard D. Lane \\ and \\ Robert W. Rhoades \\ Department of Anatomy and Neurobiology \\ Medical College of Ohio \\ 3000 Arlington Avenue \\ Toledo, $\mathrm{OH} 43614$
}

Running Title: Clorgyline’s effects on GAP-43 expression in the developing S-I

24 Text Pages

3 Figures 
*To whom correspondence should be addressed: Kay Kesterson, phone (419) 383-4109;

fax (419)383-3008; email kkesterson@mco.edu

Supported in part by DE07743

Published in Developmental Brain Research 139 (2002) 167-174 
Abstract

Elevating cortical serotonin (5-HT) in rats with clorgyline, a monoamine oxidase A $\left(\mathrm{MAO}_{\mathrm{A}}\right)$ inhibitor, from postnatal day (P-) 0 to P-6 delays the organization of thalamocortical afferent fibers into a vibrissae-related pattern in the somatosensory cortex (S-I). Despite continued elevation of cortical 5-HT, the thalamocortical fibers form a characteristic vibrissae pattern of barrels in layer IV of S-I by P-8. The growth-associated protein, GAP-43, is transiently expressed in developing S-I cortex of rats in a vibrissae related pattern until P-7. After P7, GAP-43 expression is reduced in the barrel centers and increased in the septa. The present study evaluated the effect of elevated 5-HT levels on the distribution of GAP-43 immunoreactivity in S-I. We employed 5-HT immunocytochemistry and 1,1'dioctadecyl-3,3,3”,3'- tetramethylindocarbocyanine perchlorate (DiI) labeling of thalamic radiations to confirm a "barrelless" phenotype in P-6 clorgyline-treated animals and a recovered barrel pattern in treated animals allowed to survive until P-8 and P-10. GAP43 immunocytochemistry was used to evaluate the cortical distribution of this protein in similarly treated littermates. Continuous inhibition of $\mathrm{MAO}_{\mathrm{A}}$ from $\mathrm{P}-0$ to P-6 resulted in a corresponding loss of the GAP-43 vibrissae-related pattern at P-6. Despite continued elevation of cortical 5-HT until P-8 and P-10, the characteristic vibrissae-complementary pattern of GAP-43 emerged with expression concentrated in the septa and rows. GAP-43 vibrissae-related thalamocortical axon pattern never appeared in the clorgyline-treated animals. Thus, while elevated 5-HT delays development of a vibrissae-related pattern of 
thalamocortical afferents, it does not appear to alter the time when a GAP-43 vibrissaerelated complementary pattern emerges.

Theme: Sensory Systems

Topic: Somatosensory Cortex and Thalamocortical Relationships

Keywords: clorgyline, barrel, cortical serotonin, immunocytochemistry, GAP-43 


\section{Introduction}

Serotonin (5-HT) and several of its receptor subtypes, serotonin $1 \mathrm{~B}$ receptors $\left(5-\mathrm{HT}_{1 \mathrm{~B}}\right)$ [24,3], and serotonin 2A [27], transiently appear in a mystacial vibrissae-related pattern in the somatosensory cortex (S-I) during early postnatal development $[10,13,15,23,30]$. In addition, vibrissae-related thalamocortical axons and their parent cells also transiently express the high affinity 5-HT transporter (SERT) during the perinatal period, allowing 5-HT uptake into these fibers for a restricted time period [4,23,8,27,6].

Previous research has demonstrated that altering cortical 5-HT concentrations during perinatal development disrupts the vibrissae-related pattern in the rodent S-I. Neonatal mice lacking the gene that encodes monoamine oxidase $\mathrm{A}\left(\mathrm{MAO}_{\mathrm{A}}\right)$ have significantly elevated 5-HT levels and a complete absence of vibrissae related barrels (cellular aggregates in lamina IV corresponding to each vibrissae separated by relatively cellsparse septa) in the S-I [9]. Pharmacological elevation of cortical 5-HT with a $\mathrm{MAO}_{\mathrm{A}}$ inhibitor, clorgyline, yields a dose-dependent range of disruptions in thalamocortical development. Perinatal rodents treated with clorgyline $(10 \mathrm{mg} / \mathrm{kg})$ two and three times daily yielded larger vibrissae-related barrels and higher cortical 5-HT levels compared to control animals. Four daily clorgyline injections produced significantly larger vibrissaerelated barrels with indistinct septa in the S-I and significantly augmented cortical 5-HT levels $[31,6,19]$. Increasing clorgyline injections to five times daily yielded a "barrelless" pattern in the S-I and cortical 5-HT levels > 1500\% above normal levels in rats killed on 
postnatal day (P-) 6 [6]. However, by P-8, thalamocortical axons and layer IV cells begin to reorganize and form vibrissae-related patterns despite continued elevation of cortical 5-HT levels. By P-10, thalamocortical axons and layer IV aggregates form qualitatively and quantitatively normal patches corresponding to the mystacial vibrissae $[6,19]$.

Previous studies suggest that the growth-associated protein, (GAP)-43, a nervous systemspecific phosphoprotein that binds to the plasma membrane of growth cones, is involved in the preliminary ordering and segregation of thalamocortical axons $[14,26]$. In the developing S-I, GAP-43 is transiently expressed as a vibrissae-related pattern in the cortex from P-3 to P-7 that coincides with the time-course of thalamocortical axon segregation in normal rats. After P-7, the GAP-43-positive patches in S-I disappear and the density of staining is increased in the septa between the barrels. This complementary pattern of GAP-43 persists into adulthood [14]. In mice lacking the gene encoding GAP43, thalamocortical axons do not extend to layer IV of S-I, and an ordered whiskerrelated somatotopic map never forms [26] which suggests that the role of GAP-43 is important in thalamocortical axon pathfinding. The present experiments were performed to determine if augmented 5-HT levels produced by pharmacological inhibition of $\mathrm{MAO}_{\mathrm{A}}$ from birth affects the expression of GAP-43 in the developing rat S-I. 


\section{Materials and Methods}

\section{Experimental Animals}

The animals utilized in this study were normal newborn male and female Sprague-

Dawley rats. All rats were housed in an approved animal facility and supplied with ad libitum food and water. All procedures performed in this study were approved by the Institutional Animal Care and Use Committee (IACUC) and were conducted according to NIH and USDA guidelines.

\section{Neonatal administration of clorgyline}

Clorgyline (Chemicon; $10 \mathrm{mg} / \mathrm{kg}$ ) was dissolved in sterile saline and administered subcutaneously to neonatal rat pups within 12 hours of birth (P-0). Pups were injected every 5 hours from P-0 to P-6. Pups were sacrificed after the last injection on P-6, or on P-8 or

P-10. All pups were weighed daily and the mean weight per litter calculated in order to determine the injection volume. Littermates that served as controls were injected with sterile saline on an identical schedule.

\section{Perfusion fixation}

Control and clorgyline-treated P-6, P-8 and P-10 rat pups were anesthetized with isoflurane and perfused transcardially with $0.9 \%$ saline in $0.1 \mathrm{M}$ sodium phosphate buffer 
(PBS; $\mathrm{pH} 7.4,21^{\circ} \mathrm{C}$ ) followed by $4 \%$ paraformaldehyde solution in the same buffer at $4^{\circ} \mathrm{C}$.

\section{Labeling of vibrissae-related thalamocortical afferents}

Perfused brains were removed from the skull and the cortices separated from the underlying diencephalon. Crystals of 1,1'-dioctadecyl-3,3,3”,3'tetramethylindocarbocyanine perchlorate (DiI) dissolved in 100\% methanol were painted on the thalamic radiations with a fine paintbrush and the radiations then covered with warm $4 \%$ agar. After the agar hardened, the cortices were placed in $4 \%$ paraformaldehyde and stored for two weeks. The cortices were then placed into fresh $4 \%$ paraformaldehyde and stored at $37^{\circ} \mathrm{C}$ for an additional three to four weeks. The cortices were then flattened parallel to the pial surface and $50 \mu \mathrm{m}$ sections were cut on a freezing microtome. Sections were placed in PBS, plated immediately on glass slides, coverslipped, and visualized under low power magnification with episcopic fluorescence. Labeled thalamocortical afferents were photographed immediately.

\section{Immunostaining for 5-HT}

Perfused brains were removed from the skull and post-fixed for 12 to 36 hours in $4 \%$ paraformaldehyde. The cerebral cortices flattened and cut into $50 \mu \mathrm{m}$ sections on a freezing microtome. After two rinses in PBS, sections were incubated overnight in primary antiserum directed against 5-HT (Incstar; $1: 25,000$ dilution; $21^{\circ} \mathrm{C}$ ). The primary antibody was diluted in PBS that contained $1 \%$ bovine serum albumin and $0.4 \%$ Triton 
X-100 (PBS-Tx). Sections were rinsed for 30 minutes in PBS containing PBS-Tx and then incubated for one hour in biotinylated anti-rabbit IgG (Vector Labs; 1:400 dilution in PBS-Tx). Tissue sections were again rinsed in PBS-Tx and incubated for 30 minutes in ABC complex (Vector Labs; 1:200 dilution in PBS-Tx). After a final rinse in PBS-Tx, sections were reacted in a $50.0 \mathrm{mg} \%$ solution of 3,3'-diaminobenzidine (DAB) containing $0.02 \% \mathrm{H}_{2} \mathrm{O}_{2}$. Stained sections were mounted on glass slides, allowed to air dry, dehydrated in 100\% ethanol, cleared in xylene, and coverslipped.

\section{Immunostaining for GAP-43}

Perfused brains were removed from the skull, post-fixed for 36 to 48 hours in $4 \%$ paraformaldehyde and the cerebral cortices flattened and cut into $50 \mu \mathrm{m}$ sections on a freezing microtome. After three rinses in PBS for 30 minutes, tissue sections were incubated 72 to 86 hours in primary antiserum directed against GAP-43 (Chemicon; 1:1000 dilution; $21^{\circ} \mathrm{C}$ ). The primary antibody was diluted in PBS-Tx that contained $1 \%$ bovine serum albumin, and 4\% normal goat serum (Vector Laboratories). Sections were rinsed for 30 minutes in PBS-Tx and then incubated for 24 to 36 hours in biotinylated anti-rabbit IgG (Vector Labs; 1:400 dilution in PBS-Tx). Tissue sections were again rinsed in PBS-Tx and incubated for 24 hours in ABC complex (Vector Labs; 1:100 dilution in PBS-Tx). After a final rinse in PBS-Tx, sections were reacted in a $50.0 \mathrm{mg} \%$ solution of DAB containing $0.02 \% \mathrm{H}_{2} \mathrm{O}_{2}$. Stained sections were mounted on glass slides, allowed to air dry, dehydrated in 100\% ethanol, cleared in xylene, and coverslipped. 


\section{Results}

\section{Effects of clorgyline treatment on the patterning of DiI-labeled thalamocortical axons and on the expression of 5-HT, and GAP-43.}

The thalamocortical afferent patterns of saline-treated P-6, 8, and 10 rat pups (Fig. 1C, 2C and 3C respectively) reflect the typical pattern of vibrissae-related DiI-labeled afferents in the S-I. DiI-labeling of thalamocortical afferents in a P-6 clorgyline-treated pup demonstrates fused barrels and rows in the S-I with no discernible segmentation of patches corresponding to the mystacial vibrissae (Fig. 1D). In animals treated with clorgyline and killed on P-8, segmentation of the thalamocortical afferents is visible (Fig. 2D). DiI-labeling of thalamocortical afferents in clorgyline-treated animals killed on P10 reveal an almost completely recovered pattern of vibrissae-related afferents in the S-I (Fig. 3D). These results are also consistent with prior findings from this laboratory $[6,19]$.

Treatment with clorgyline from P-0 through P-6 (Fig. 1B) resulted in a complete loss of the 5-HT vibrissae-related pattern normally observed in S-I of rats at P-6 (Fig. 1A). By P-8, the 5-HT-stained sections of clorgyline-treated animals demonstrated discernible barrels (Fig. 2B). However, representation of the posteromedial barrel subfield (PMBSF) is not as clear as the 5-HT stained sections of the saline-treated animals (Fig. 2A). By P10, the 5-HT stained sections of clorgyline-treated animals (Fig. 3B) demonstrate a recovered barrel pattern similar to saline-treated animals (Fig. 3A). 
Focal patches of GAP-43 staining corresponding to the mystacial vibrissae were clearly evident in saline-treated P-6 rat pups (Fig. 1E). In contrast to the saline-treated animals, the GAP-43 immunostained sections of P-6 rat pups that were treated with clorgyline did not demonstrate a vibrissae-related pattern (Fig. 1F). The S-I sections showed intense GAP-43 staining but with no discernible barrel field or demarcation of the head representation as seen in the DiI-labeled and 5-HT immunostained sections of P-6 clorgyline treated rats (Fig.s 1B,D). The distribution of GAP-43 immunoreactivity in the cortices of saline and clorgyline-treated animals killed on P-8 was similar. Relative to the normal P-6 GAP-43 pattern, there was a decreased expression of GAP-43 in the barrel centers and enhanced staining in the septal regions between the vibrissa rows as well as in the surrounding dysgranular cortex (Fig.s 2E and 2F). Immunostained GAP-43 sections of P-10 saline-treated animals demonstrated a complementary pattern to that of DiI-labeled or 5-HT stained sections. The barrels were void of any expression of GAP43 while the septa displayed GAP-43 staining (Fig. 3E). Barrels of clorgyline-treated pups killed on P-10 were similarly void of any immunostaining for GAP-43 while the expression of the protein is clearly present in the septa (Fig. 3F). All sections from clorgyline-treated P-8 and 10 pups displayed less GAP-43 immunoreactivity than salinetreated P-8 and 10 animals. 


\section{DISCUSSION}

The disruption of the thalamocortical afferent vibrissa pattern in S-I of P-6 rats produced by clorgyline mediated elevation in 5-HT as well as the subsequent recovery of the pattern at P-8 and P-10 as demonstrated by DiI-labeling and 5-HT immunostaining in this study (Fig.s 1A-D, 2A-D, 3A-D) are consistent with prior results from studies in both mice and rats $[9,3,6,33,19]$. Similarly, the GAP-43 immunostained vibrissae pattern observed in S-I of P-6 normal rats was disrupted by clorgyline treatment (Fig.s 1E,F). However, unlike the DiI-labeled thalamocortical afferents and 5-HT immunostained fibers that were localized to S-I head representation, the disrupted P-6 GAP-43 pattern was uniformly distributed across the S-I cortex. By P-8 and P-10, the GAP-43 immunostaining pattern observed in clorgyline treated rats (Fig.s 2F and 3F, respectively) was similar to that of the age-matched controls (Fig.s 2E and 3E), and consisted of a septal pattern complimentary to the normal thalamocortical vibrissae pattern.

Thalamocortical afferents originating from rat ventrobasal thalamic nucleus (VB) have been indicated as the source of the GAP-43 transiently expressed during the first postnatal week. This is based on the observation that cauterization of row $\mathrm{C}$ whiskers on P-0 converted the GAP-43 immunostaining pattern from the normal parcelated row C barrels into a continuous band [14]. The VB cells provide the thalamocortical afferent input to the cortical plate that organizes into a vibrissae-related pattern by P-2 [18]. This 
organization precedes the appearance on P-3 of the vibrissae related GAP-43 pattern in the S-I of normal rats [14].

The redistribution of GAP-43 immunoreactivity after P-7 to septal and disgranular regions of S-I while DiI labeling and 5-HT immunocytochemistry continue to define the vibrissae-related pattern associated with the VB thalamocortical afferents indicates that a different population of neurons or neuronal fibers takes over expression of GAP-43. The origin of the GAP-43 positive axons in the septa has not been determined. However, Erzurumulu et al. [14] suggests neurons located in the rostral portion of the thalamic posterior complex (POm) are a likely source. The POm afferents form a complementary pattern in layer IV of S-I similar to the septal GAP-43 pattern demonstrated in normal P7 rats $[20,12,25]$. Another possible source suggested by Erzurumulu et al. [14] for the P7 GAP-43 positive septal fibers are the callosal projections of S-I [32,2,28]. Over the course of the first postnatal week, the widespread distribution of callosal projection neurons throughout the entire somatosensory cortex is almost completely eliminated from the areas in the SI innervated by the vibrissae-related thalamocortical axons $[16,17]$. By P-7, the callosal projections form a complementary pattern that surrounds individual barrels corresponding to the vibrissae and sinus hairs $[28,21]$. While these projections are directed primarily to layers III, V, and VI in the adult, their distribution in postnatal animals has not been well defined and hence could include layer IV. The emergence of the complementary pattern delineated by the callosal fibers parallels the appearance of GAP-43 complementary pattern on P-7. 
The specific function of GAP-43 in the development of thalamocortical patterns is unknown. In vivo studies have shown that GAP-43 is present in growth cones and is known to regulate the growth of developing axons in response to extracellular signals [5]. Increasing expression of GAP-43 in vivo [1] produces ectopic sprouting at neuromuscular junctions and in the terminal field of hippocampal mossy fibers. The importance of GAP-43 in the normal development of S-I is apparent from a study by Maier et al. [26] that showed mice lacking theGAP-43 gene fail to develop an orderly vibrissa pattern and the thalamocortical afferents project abnormally in S-I. In these same animals, the vibrissae-related patterns in the trigeminal brainstem nuclei are normal while formation of barraloids in the thalamus is only partially affected. Whether the increased expression of GAP-43 in the cortices of developing rats with elevated serotonin levels is in some degree responsible for loss of pattern at P-6, perhaps by promoting exuberant growth of the POm thalamocortical fibers remains to be determined.

The most striking effect of elevated levels of serotonin noted in this study was the conversion of barrel specific GAP-43 immunoreactive staining pattern of a P-6 normal animal to the uniformly intense GAP-43 immunoreactivity seen throughout the layer IV cortical section in the P-6 clorgyline treated animal. A similar increase in GAP-43 expression in the corpus callosum and hippocampus of P-6 rats was noted in cocainetreated prenatal rats [11]. Cocaine is a monoamine reuptake inhibitor which elevates extracellular levels of serotonin when given acutely $[7,29]$. One explanation of our 
observed effect is that the elevated serotonin induces a premature GAP-43 expression by fibers in the septa and dysgranular cortex, which along with the GAP-43 positive unpatterned VB thalamocortical fibers produce uniform cortical staining. However, it seems unlikely that the distribution of GAP-43 in the septal and dysgranular regions would so closely match that of the thalamocortical fibers irregularly distributed in the S-I head region that complete masking would be achieved. It seems more likely that the elevated serotonin has had the added effect of downregulating the expression of GAP-43 in the VB thalamocortical fibers, which while no longer organized into the vibrissae pattern, are still localized within the head region (Fig. 1D). This would account for the observation that no head region borders are apparent in the GAP-43 immunostained sections from clorgyline treated animals (Fig. 1F).

Another unexpected result is that a normal-like GAP-43 pattern appears in P-8 clorgyline-treated animals, coinciding with the recovery of the DiI and 5-HT labeled vibrissae-related pattern in S-I. If as suggested above, the GAP-43 pattern in clorgyline treated animals primarily reflect the distribution of septal fibers, then this finding would suggest that these fibers organize into a septal pattern by P-8. This septal organization is supported by a previous study of examining the effects of clorgyline induced elevation of cortical 5-HT upon the intracortical projections in layer IV of rat S-I which also normally distribute primarily within the septa [22]. Neonatal rats chronically injected with clorgyline from birth until P-6 lack the normal intracortical fiber septal pattern on P-6; 
however, recovery of the pattern complimentary to the barrels occurs when the animals are allowed to survive until P-10.

In conclusion, our results suggest that the effects of elevating cortical 5-HT levels via pharmacological inhibition of $\mathrm{MAO}_{\mathrm{A}}$, affects the early ( $<$ P-8) expression of GAP-43 in layer IV of S-I. The data suggests that increasing cortical 5-HT does not lead to a general developmental delay, but rather, may accelerate development of GAP-43 expressing axons targeting the septal regions. 


\section{Acknowledgements}

The authors would like to gratefully thank Rebecca Wynn and Asad Shah for assistance with injections. We would also like to thank Rebecca Wynn and Dawn Pauli for technical assistance. 


\section{References}

[1] L. Aigner, S. Arber, J.P. Kapfhammer, T. Laux, C. Schneider, F. Botteri, H.R. Brenner, P. Caroni, Overexpression of the neural growth-associated protein GAP-43 induces nerve sprouting in the adult nervous system of transgenic mice, Cell 83 (1995) 269-278.

[2] R.M. Akers, H.P. Killackey, Organization of corticocortical connections in the parietal cortex of the rat, J. Comp. Neurol. 181 (1978) 513-537

[3] C.B. Bennett-Clarke, M.J. Leslie, N.L. Chiaia, R.W. Rhoades, Serotonin $1 \mathrm{~B}$ receptors in the developing somatosensory and visual cortices are located on thalamocortical axons, Proc. Natl. Acad. Sci. USA 90 (1993) 153-157.

[4] C.B. Bennett-Clarke, N.L. Chiaia, R.W. Rhoades, Thalamocortical afferents in rat transiently express high affinity serotonin uptake sites, Brain Res. 733 (1996) 301306.

[5] L.I. Benowitz, A. Routtenberg, GAP-43: an intrinsic determinant of neuronal development and plasticity, Trends Neurosci. 20 (1997) 84-91. 
[6] C.B. Boylan, C.A. Bennett-Clarke, R. S. Crissman, R. D. Mooney, R.W. Rhoades, Clorgyline treatment elevates cortical serotonin and temporarily disrupts the vibrissae-related pattern in rat somatosensory cortex, J. Comp. Neurol. 427 (2000) 139-149.

[7] C.W. Bradberry, J.B. Nobiletti, J.D. Elsworth, B. Murphy, P. Jatlow, R.H. Roth, Cocaine and cocaethylene: microdialysis comparison of brain drug levels and effects on dopamine and serotonin, J. Neurochem. 60 (1993) 1429-1435.

[8] G. Bruning, O. Liangos, Transient expression of the serotonin transporter in the developing mouse thalamocortical system, Acta Histochem. 99 (1997) 117-121.

[9] O. Cases, T. Vitalis, I. Seif, E. De Maeyer, C. Sotelo, P. Gaspar, Lack of barrels in the somatosensory cortex of monoamine oxidase A-deficient mice: role of a serotonin excess during the critical period, Neuron 16 (1996) 297-307.

[10] N.L. Chiaia, S.E. Fish, W.R. Bauer, B.A. Figley, M. Eck, C.A. Bennett-Clarke, R.W. Rhoades, Effects of postnatal blockade of cortical activity with tetrodoxtoxin upon the development and plasticity of vibrissae-related patterns in the somatosensory cortex hamsters, Somat. \& Motor Res. 11 (1994) 219-228. 
[11] C.K. Clarke, J. Muneyyirci, E. Azmitia, P.M. Whitaker-Azmitia, Prenatal cocaine delays astroglial maturation: immunodensitometry shows increased markers of immaturity (vimentin and GAP-43) and decreased proliferation and production of the growth factor S-100, Dev. Brain Res. 91 (1996) 268-273.

[12] J. Chmielowska, G.E. Carvell, D.J. Simons, Spatial Organization of thalamocortical and corticothalamic projection systems in the rat SmI barrel cortex, J. Comp. Neurol. 285 (1989) 325-338.

[13] R.J. D’Amato, M.E. Blue, B.L. Largent, D.R. Lynch, D.J. Ledbetter, M.E. Molliver, S.H. Snyder, Ontogeny of the serotonergic projection to rat neocortex: transient expression of a dense innervation to primary sensory areas, Proc. Natl. Acad. Sci. USA 84 (1987) 4322-4326.

[14] R.S. Erzurumlu, S. Jhaveri, L.I. Benowitz, Transient patterns of GAP-43 expression during the formation of barrels in the rat somatosensory cortex, J. Comp. Neurol. 292 (1990) 443-456. 
[15] M. Fujimiya, H. Kimura, T. Maeda, Postnatal development of serotonin nerve fibers in the somatosensory cortex of mice studied by immunohistochemistry, J. Comp. Neurol. 246 (1986) 191-201.

[16] G.O. Ivy, R. M. Akers, H.P. Killackey, Differential distribution of callosal projection neurons in the neonatal and adult rat, Brain Res. 173 (1979) 532-537.

[17] G.O. Ivy, H.P. Killackey, The ontogeny of the distribution of callosal projection neurons in the rat parietal cortex, J. Comp. Neurol. 195 (1981) 367-389.

[18] G.O. Ivy, H.P. Killackey, Ontogenetic changes in the projections of neocortical neurons, J. Neurosci. 2 (1982) 735-743.

[19] K.L. Kesterson, C.B. Boylan, C.L. Young-Davies, C.A. Bennett-Clarke, R.D. Lane, R.W. Rhoades, Timing and clorgyline dose are important for disrupting the vibrissae-related pattern in rat somatosensory cortex, (2001) (in preparation).

[20] K.A. Koralek, K.F. Jensen, H.P. Killackey, Evidence for two complementary patterns of thalamic input to the rat somatosensory cortex, Brain Res. 463 (1988) 346351. 
[21] K.A. Koralek, H.P. Killackey, Callosal projections in rat somatosensory cortex are altered by early removal of afferent input, Proc. Natl. Acad. Sci. USA 87 (1990) 1396-1400.

[22] R.D. Lane, T. Rizk, N.L. Chiaia, R.D. Mooney, R.W. Rhoades, Effects of alterations of the vibrissae-related organization of thalamocortical axons upon the organization and outgrowth of intracortical connections in the barrelfield of the rat, Somat. \& Motor Res. (2002) (In Press).

[23] C. Lebrand, O. Cases, C. Adelbrecht, A. Doye, C. Alvarez, S. El Mestikawy, I. Seif, P. Gaspar, Transient uptake and storage of serotonin in developing thalamic neurons, Neuron 17 (1996) 823-835.

[24] M.J. Leslie, C.A. Bennett-Clarke, R.W. Rhoades, Serotonin-1B receptors for a transient vibrissa-related pattern in the primary somatosensory cortex of the developing rat, Dev. Brain. Res. 69 (1992) 143-148. 
[25] S.M. Lu, R.C. Lin, Thalamic afferents of the rat barrel cortex: a light- and electron- microscopic study using Phaseolus vulgaris leucoagglutinin as an anterograde tracer, Somotosens. Mot. Res. 10 (1993) 1-16.

[26] D.L. Maier, M. Shyamala, S.L. Donovan, D. Soppet, L. Tessarollo, J.S. McCasland, K. F. Meiri, Disrupted cortical map and the absence of cortical barrels in growth-associated protein (GAP)-43 knockout mice, Proc. Natl. Acad. Sci. 96 (1999) 9397-0402.

[27] S. Mansour-Robaey, N. Mechawar, F. Radja, C. Beaulieu, L. Descarries, Quantified distribution of serotonin transporter and receptors during the postnatal development of the rat barrel field cortex, Dev. Brain Res. 107 (1998) 159-163.

[28] J. Olavarria, R.C. Van Sluyters, H.P. Killackey, Evidence for the complementary organization of callosal and thalamic connections within the rat somatosensory cortex, Brain Res. 291 (1984) 364-368.

[29] M.E. Reith, M.Y. Li, Q.S. Yan, Extracellular dopamine, norepinephrine, and serotonin in the ventral tegmental area and nucleus accumbens of freely moving rats 
during intracerebral dialysis following systemic administration of cocaine and other uptake blockers, Psychopharm. 134 (1997) 309-317.

[30] R.W. Rhoades, C.A. Bennett-Clarke, N.L. Chiaia, F.A. White, G.L. McDonald, J.H. Haring, M.F. Jacquin, Development and lesion induced reorganization of the cortical representation of the rat's body surface as revealed by immunocytochemistry for serotonin, J. Comp. Neurol. 293 (1990) 190-207.

[31] T. Vitalis, O. Cases, J. Callebert, J. Launay, J. Price, I. Seif, P. Gaspar, Effect of monoamine oxidase A inhibition on barrel formation in the mouse somatosensory cortex: determination of a sensitive period, J. Comp. Neurol. 393 (1998) 169-184.

[32] S.P. Wise, E.G. Jones, The organization and postnatal development of the commissural projection of the rat somatic sensory cortex, J. Comp. Neurol. 168 (1976) 313-343.

[33] C.L. Young-Davies, C.A. Bennett-Clarke, R.D. Lane and R.W. Rhoades, Selective facilitation of the serotonin (1B) receptor causes disorganization of thalamic afferents and barrels in somatosensory cortex of rat, J. Comp. Neurol. 425 (2000) 130-138. 
Figure 1. Serotonin immunoreactivity, DiI-labeled patterns of thalamocortical afferents, and GAP-43 immunoreactivity in flattened sections from P-6 rats that was either injected with saline or clorgyline. A, C, and E: show the characteristic normal pattern of 5-HT immunostaining, DiI-labeled thalamocortical afferents, and GAP-43 immunostaining respectively in saline-treated rats. B: shows the pattern of 5-HT immunostaining in an animal that was treated with clorgyline from birth. D: shows the DiIlabeled pattern of thalamocortical afferents in a clorgyline-treated animal. Note the lack of segmentation of vibrissae-related patches in Figures 1B and D. F: shows a GAP-43 immunostained section through layer IV of S-I in an animal treated with clorgyline. In addition to the lack of segmented vibrissae-related patches, the borders of the head region seen it the DiI-labeled thalamocortical afferents of clorgyline treated rat (Fig. 1D) is also absent. Scale bar $=1 \mathrm{~mm}$. 


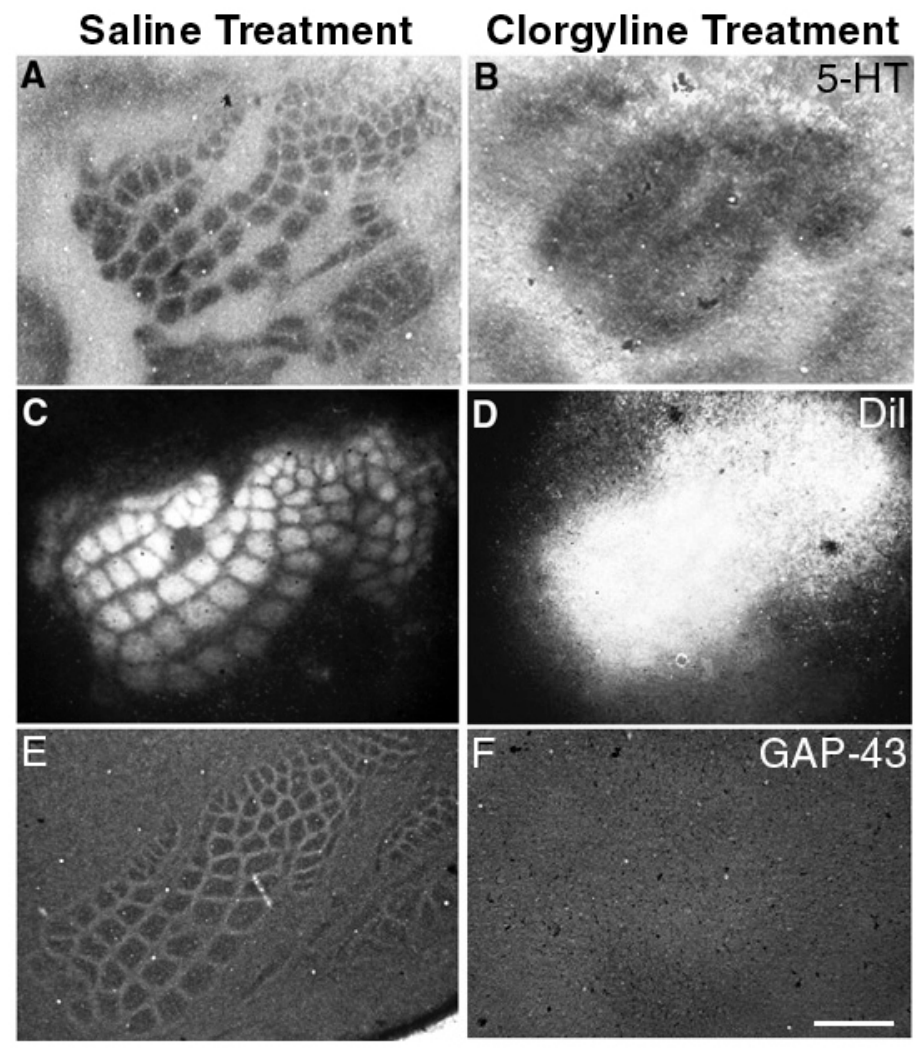


Figure 2. Serotonin immunoreactivity, DiI-labeled patterns of thalamocortical afferents, and GAP-43 immunoreactivity in flattened sections from P-8 rats that was either injected with saline or clorgyline from birth to P-6. A, C, and E: show the typical pattern of 5-HT immunostaining, DiI-labeled thalamocortical afferents, and GAP-43 immunostaining respectively in saline-treated rats. B and D: show a 5-HT immunostained section and the DiI-labeled thalamocortical afferents (respectively) in clorgyline-treated animals. Segmentation of vibrissae-related patches in Figures 2B and D is present; however, the septa appears less distinct than in the sections of the saline-treated rats. F: shows a GAP-43 immunostained section through layer IV of S-I in an animal treated with clorgyline. Lightly stained vibrissae-related patches are demonstrated with increased GAP-43 immunoreactivity in the septa. Scale bar $=1 \mathrm{~mm}$. 


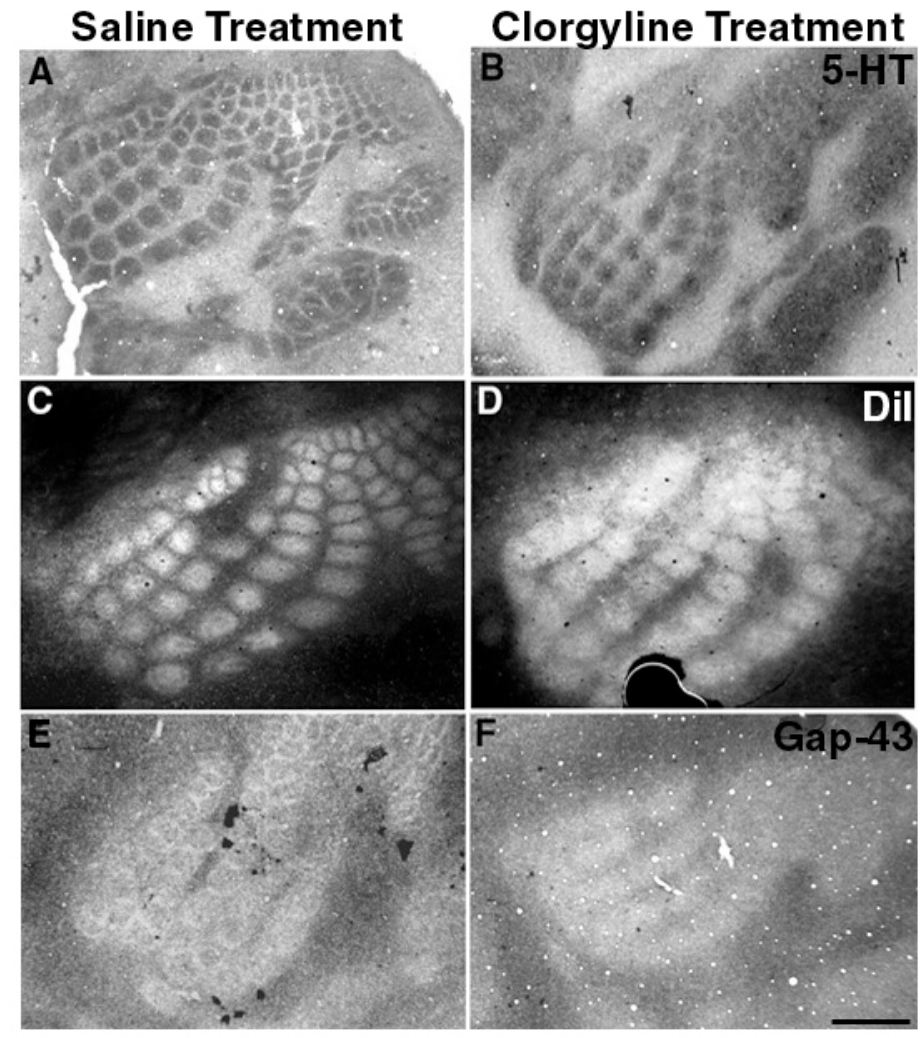


Figure 3. Serotonin immunoreactivity, DiI-labeled patterns of thalamocortical afferents, and GAP-43 immunoreactivity in flattened sections from P-10 rats that was either injected with saline or clorgyline from birth to P-6. A, C, and E: show the characteristic pattern of 5-HT immunostaining, DiIlabeled thalamocortical afferents, and GAP-43 immunostaining respectively in saline-treated rats. B and D: show a 5-HT immunostained section and the DiI-labeled thalamocortical afferents (respectively) in clorgyline-treated animals. Typical, normal-like vibrissae-related patterns are shown in B and D. F: shows a GAP-43 immunostained section representative of P-10 animals treated with clorgyline. Note that the vibrissae-related patches are not immunostained for GAP-43 while the septa displays GAP-43 immunoreactivity very similar in appearance to the GAP-43 immunostaining present in the P-10 controls $(\mathrm{E})$. Scale bar $=1$ mm. 


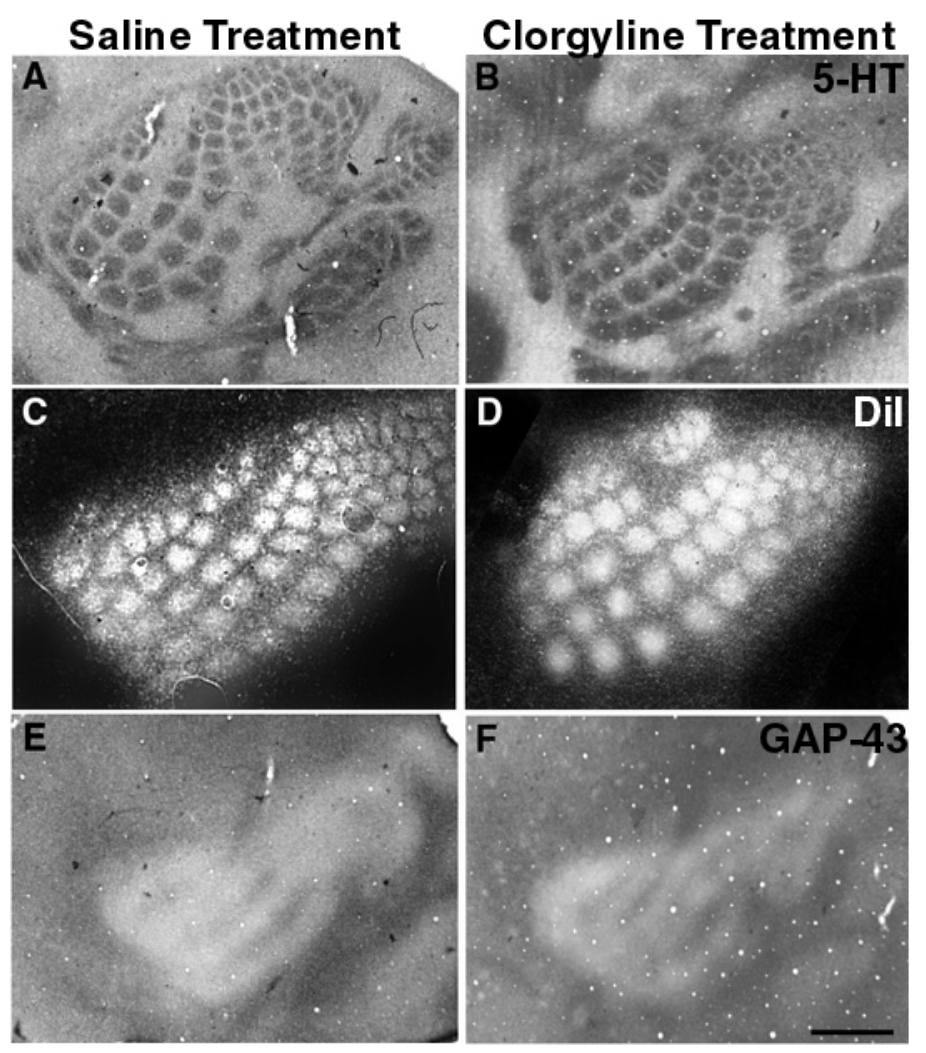




\section{REFERENCES}

Aigner, L.; Arber, S.; Kapfhammer, J.P.; Laux, T.; Schneider, C.; Botteri,F.; Brenner, H.R.; and Caroni, P. (1995) Overexpression of the neural growth-associated protein GAP-43 induces nerve sprouting in the adult nervous system of transgenic mice. Cell 83, 269-278.

Akers, R.M.; and Killackey, H.P. (1978) Organization of corticocortical connections in the parietal cortex of the rat. J. Comp. Neurol. 181, 513-537.

Bennett-Clarke C.B.; Chiaia, N.L.; and Rhoades, R.W. (1996) Thalamocortical afferents in rat transiently express high affinity serotonin uptake sites. Brain Res. 733, 301306.

Bennett-Clarke, C.B.; Leslie, M.J.; Chiaia, N.L.; and Rhoades, R.W. (1993) Serotonin 1 B receptors in the developing somatosensory and visual cortices are located on thalamocortical axons. Proc. Natl. Acad. Sci. USA 90, 153-157.

Bendotti, C.; Servadio, A.; and Samanin, R. (1991) Distribution of Gap-43 mRNA in the brain stem of adult rats as evidenced by in situ hybridization: localization within monaminergic neurons. J Neurosci. 11, 600-7. 
Benowitz, L.I; and Routtenberg, A. (1997) GAP-43: an intrinsic determinant of neuronal development and plasticity. Trends Neurosci. 20 84-91.

Boylan C.B.; Bennett-Clarke C.A.; Chiaia N.L., and Rhoades R.W. (2000a) Time course of expression and function of the serotonin transporter in the neonatal rat's primary somatosensory cortex. Somatosens Mot Res.17, 52-60.

Boylan, C.B.; Bennett-Clarke, C.A.; Crissman, R. S.; Mooney, R. D.; and Rhoades, R.W. (2000b) Clorgyline treatment elevates cortical serotonin and temporarily disrupts the vibrissae-related pattern in rat somatosensory cortex, J. Comp. Neurol. 427, 139-149.

Bradberry, C.W.; Nobiletti, J.B.; Elsworth, J.D.; Murphy, B.; Jatlow, P.; and Roth, R.H. (1993) Cocaine and cocaethylene: microdialysis comparison of brain drug levels and effects on dopamine and serotonin. J. Neurochem. 60, 1429-1435.

Bruning, G.; and Liangos, O. (1997) Transient expression of the serotonin transporter in the developing mouse thalamocortical system. Acta Histochem. 99, 117-121.

Cases, O.; Seif, I.; Grimsby, J.; Gaspar, P.; Chen, K.; Pournin, S; Muller, U.; Aguet, M.; Babinet, C ; Shih, J.C.; and De Maeyer, E. (1995) Aggressive behavior and 
altered amounts of brain serotonin and norepinephrine in mice lacking MAOA. Science 268, 1763-6.

Cases, O.; Vitalis, T.; Seif, I.; De Maeyer, E.; Sotelo, C.; and Gaspar, P. (1996) Lack of barrels in the somatosensory cortex of monoamine oxidase A-deficient mice: role of a serotonin excess during the critical period. Neuron 16, 297-307.

Chiaia, N.L.; Fish, S.E.; Bauer, W.R.; Figley, B.A.; Eck, M.; Bennett-Clarke, C.A.; and Rhoades, R.W. (1994) Effects of postnatal blockade of cortical activity with tetrodoxtoxin upon the development and plasticity of vibrissae-related patterns in the somatosensory cortex hamsters. Somat. \& Motor Res. 11, 219-228.

Clarke, C.K.; Muneyyirci, J.; Azmitia, E.; and Whitaker-Azmitia, P.M. (1996) Prenatal cocaine delays astroglial maturation: immunodensitometry shows increased markers of immaturity (vimentin and GAP-43) and decreased proliferation and production of the growth factor S-100. Dev. Brain Res. 91, 268-273.

Ching, Y.P.; Averill, S.; Wilkin, GP.; Wotherspoon, G.; Priestley, J. V. (1994) Serotonergic terminals express a growth associated protein (GAP-43) in the adult rat spinal cord. Neurosci. Lett. 167, 67-72. 
Chmielowska, J.; Carvell, G.E.; and Simons, D.J. (1989) Spatial Organization of thalamocortical and corticothalamic projection systems in the rat SmI barrel cortex. J. Comp. Neurol. 285, 325-338.

D’Amato, R.J.; Blue, M.E.; Largent, B.L.; Lynch, D.R.; Ledbetter, D.J.; and Molliver, M.E.; and Snyder, S.H. (1987) Ontogeny of the serotonergic projection to rat neocortex: transient expression of a dense innervation to primary sensory areas. Proc. Natl. Acad. Sci. USA 84, 4322-4326.

Erzurumlu, R.S.; Jhaveri, S.; and Benowitz, L.I. (1990) Transient patterns of GAP-43 expression during the formation of barrels in the rat somatosensory cortex. J. Comp. Neurol. 292, 443-456.

Fujimiya, M.; Kimura, H.; and Maeda, T. (1986) Postnatal development of serotonin nerve fibers in the somatosensory cortex of mice studied by immunohistochemistry. J. Comp. Neurol. 246 191-201.

Ivy, G.O.; Akers, R. M.; and Killackey, H.P.; (1979) Differential distribution of callosal projection neurons in the neonatal and adult rat. Brain Res. 173, 532-537.

Ivy, G.O.; and Killackey, H.P. (1981) The ontogeny of the distribution of callosal projection neurons in the rat parietal cortex. J. Comp. Neurol. 195, 367-389. 
Ivy, G.O.; and Killackey, H.P. (1982) Ontogenetic changes in the projections of neocortical neurons. J. Neurosci. 2, 735-743.

Kesterson, K.L.; Boylan, C.B.; Young-Davies, C.L.; Bennett-Clarke, C.A.; Lane, R.D.; and Rhoades, R.W. (2005) Timing and clorgyline dose are important for disrupting the vibrissae-related pattern in rat somatosensory cortex, (accepted with revisions).

Koralek, K.A.; Jensen, K.F.; and Killackey, H.P. (1988) Evidence for two complementary patterns of thalamic input to the rat somatosensory cortex. Brain Res. 463, 346-351.

Koralek, K.A.; and Killackey, H.P. (1990) Callosal projections in rat somatosensory cortex are altered by early removal of afferent input. Proc. Natl. Acad. Sci. USA 87, 1396-1400.

Lauder, J.M. (1983) Hormonal and humoral influences on brain development. Psychoneuroendocrinology 8, 121-155.

Lane, R.D.; Rizk, T.; Chiaia, N.L.; Mooney, R.D.; and Rhoades, R.W. (2002) Effects of alterations of the vibrissae-related organization of thalamocortical axons upon the 
organization and outgrowth of intracortical connections in the barrelfield of the rat, Somatosens Mot Res.19, 125-129.

Lebrand, C.; Cases, O.; Adelbrecht, C.; Doye, A.; Alvarez, C.; El Mestikawy, S.; Seif, I.; and Gaspar, P. (1996) Transient uptake and storage of serotonin in developing thalamic neurons. Neuron 17, 823-835.

Leslie M.J.; Bennett-Clarke, C.A.; and Rhoades, R.W. (1992) Serotonin-1B receptors for a transient vibrissa-related pattern in the primary somatosensory cortex of the developing rat. Dev. Brain. Res. 69, 143-148.

Lu, S.M.; and Lin, R.C. (1993) Thalamic afferents of the rat barrel cortex: a light- and electron- microscopic study using Phaseolus vulgaris leucoagglutinin as an anterograde tracer. Somotosens. Mot. Res. 10, 1-16.

Macchi, G. (1983) Old and new anotomo-functional criteria in the subdivision of the thalamic nuclei. In: Somatosensory Integration in the Thalamus. Elsevier, Amsterdam, pp.3-16.

Maier, D.L.; Shyamala, M.; Donovan, S.L.; Soppet, D.; Tessarollo, L.; McCasland, J.S.; and Meiri, K. F. (1999) Disrupted cortical map and the absence of cortical barrels 
in growth-associated protein (GAP)-43 knockout mice. Proc. Natl. Acad. Sci. 96, 9397-0402.

Mansour-Robaey, S.; Mechawar, N.; Radja, F.; Beaulieu, C.; and Descarries, L. (1998)Quantified distribution of serotonin transporter and receptors during the postnatal development of the rat barrel field cortex. Dev. Brain Res. 107, 159163.

Olavarria, J.; Van Sluyters, R.C.; and Killackey, H.P. (1984) Evidence for the complementary organization of callosal and thalamic connections within the rat somatosensory cortex. Brain Res. 291, 364-368.

Reith, M.E.; Li, M.Y.; and Yan Q.S. (1997) Extracellular dopamine, norepinephrine, and serotonin in the ventral tegmental area and nucleus accumbens of freely moving rats during intracerebral dialysis following systemic administration of cocaine and other uptake blockers. Psychopharm. 134, 309-317.

Rhoades, R.W.; Bennett-Clarke, C.A.; Chiaia, N.L.; White, F.A.; McDonald, G.L.; Haring, J.H.; and Jacquin, M.F. (1990) Development and lesion induced reorganization of the cortical representation of the rat's body surface as revealed by immunocytochemistry for serotonin. J. Comp. Neurol. 293, 190-207. 
Vitalis, T.; Cases, O.; Callebert, J.; Launay, J.; Price, J.; Seif, I.; and Gaspar, P. (1998) Effect of monoamine oxidase A inhibition on barrel formation in the mouse somatosensory cortex: determination of a sensitive period. J. Comp. Neurol. $393,169-184$.

Wise, S.P.; and Jones, E.G. (1976) The organization and postnatal development of the commissural projection of the rat somatic sensory cortex. J. Comp. Neurol. 168, 313-343.

Woolsey, T.A. (1967) Somatosensory, auditory, and visual cortical areas of the mouse. Johns Hopkins Med. J. 121, 91-112.

Woolsey, T.A.; and Van der Loos, H. (1970) The structural organization of layer IV in the somatosensory region, DI, of mouse cerebral cortex. Brain Res. 17, 205-242.

Young-Davies, C.L.;. Bennett-Clarke, C.A; Lane R.D.; and Rhoades, R.W. (2000) Selective facilitation of the serotonin (1B) receptor causes disorganization of thalamic afferents and barrels in somatosensory cortex of rat. J. Comp. Neurol. 425, $130-138$ 


\begin{abstract}
Elevating cortical serotonin (5-HT) in rats with clorgyline, a monoamine oxidase A $\left(\mathrm{MAO}_{\mathrm{A}}\right)$ inhibitor, from postnatal day (P-) 0 to P-6 delays the organization of thalamocortical afferent fibers into a vibrissae-related pattern in the somatosensory cortex (S-I). Despite continued elevation of cortical 5-HT, the thalamocortical fibers form a characteristic vibrissae pattern of barrels in layer IV of S-I by P-8. The growthassociated protein, GAP-43, is transiently expressed in developing S-I cortex of rats in a vibrissae related pattern until P-7. After P-7, GAP-43 expression is reduced in the barrel centers and increased in the septa. The present study evaluated the effect of elevated 5HT levels on the distribution of GAP-43 immunoreactivity in S-I. We employed 5-HT immunocytochemistry and 1,1'-dioctadecyl-3,3,3”,3'- tetramethylindocarbocyanine perchlorate (DiI) labeling of thalamic radiations to confirm a "barrelless” phenotype in P6 clorgyline-treated animals and a recovered barrel pattern in treated animals allowed to survive until P-8 and P-10. GAP-43 immunocytochemistry was used to evaluate the cortical distribution of this protein in similarly treated littermates. Continuous inhibition of $\mathrm{MAO}_{\mathrm{A}}$ from P-0 to P-6 resulted in a corresponding loss of the GAP-43 vibrissaerelated pattern at P-6. Despite continued elevation of cortical 5-HT until P-8 and P-10, the characteristic vibrissae-complementary pattern of GAP-43 emerged with expression concentrated in the septa and rows. GAP-43 vibrissae-related thalamocortical axon pattern never appeared in the clorgyline-treated animals. Thus, while elevated 5-HT delays development of a vibrissae-related pattern of thalamocortical afferents, it does not
\end{abstract}


appear to alter the time when a GAP-43 vibrissae-related complementary pattern emerges. 